\title{
Video Article \\ Biomolecular Detection employing the Interferometric Reflectance Imaging Sensor (IRIS)
}

Carlos A. Lopez ${ }^{1}$, George G. Daaboul ${ }^{2}$, Sunmin Ahn ${ }^{2}$, Alexander P. Reddington ${ }^{1}$, Margo R. Monroe ${ }^{2}$, Xirui Zhang ${ }^{2}$, Rostem J. Irani ${ }^{3}$, Chunxiao Yu ${ }^{4,5}$, Caroline A. Genco ${ }^{4,5}$, Marina Cretich ${ }^{6}$, Marcella Chiari ${ }^{6}$, Bennett B. Goldberg ${ }^{1}$, John H. Connor ${ }^{5}$, M. Selim Ünlü ${ }^{1,2}$

${ }^{1}$ Department of Electrical and Computer Engineering, Boston University

${ }^{2}$ Department of Biomedical Engineering, Boston University

${ }^{3}$ Center for Advanced Genomics Technology, Boston University

${ }^{4}$ Department of Medicine, Section of Infectious Diseases, Boston University School of Medicine

${ }^{5}$ Department of Microbiology, Boston University School of Medicine

${ }^{6} \mathrm{CNR}$ (National Research Council), Istituto di Chimica del Riconoscimento Molecolare

Correspondence to: M. Selim Ünlü at selim@bu.edu

URL: https://www.jove.com/video/2694

DOI: doi:10.3791/2694

Keywords: Bioengineering, Issue 51, Interferometry, label-free, biosensing, microarray, quantification, real-time detection

Date Published: 5/3/2011

Citation: Lopez, C.A., Daaboul, G.G., Ahn, S., Reddington, A.P., Monroe, M.R., Zhang, X., Irani, R.J., Yu, C., Genco, C.A., Cretich, M., Chiari, M., Goldberg, B.B., Connor, J.H., Ünlü, M.S. Biomolecular Detection employing the Interferometric Reflectance Imaging Sensor (IRIS). J. Vis. Exp. (51), e2694, doi:10.3791/2694 (2011).

\section{Abstract}

The sensitive measurement of biomolecular interactions has use in many fields and industries such as basic biology and microbiology, environmental/agricultural/biodefense monitoring, nanobiotechnology, and more. For diagnostic applications, monitoring (detecting) the presence, absence, or abnormal expression of targeted proteomic or genomic biomarkers found in patient samples can be used to determine treatment approaches or therapy efficacy. In the research arena, information on molecular affinities and specificities are useful for fully characterizing the systems under investigation.

Many of the current systems employed to determine molecular concentrations or affinities rely on the use of labels. Examples of these systems include immunoassays such as the enzyme-linked immunosorbent assay (ELISA), polymerase chain reaction (PCR) techniques, gel electrophoresis assays, and mass spectrometry (MS). Generally, these labels are fluorescent, radiological, or colorimetric in nature and are directly or indirectly attached to the molecular target of interest. Though the use of labels is widely accepted and has some benefits, there are drawbacks which are stimulating the development of new label-free methods for measuring these interactions. These drawbacks include practical facets such as increased assay cost, reagent lifespan and usability, storage and safety concerns, wasted time and effort in labelling, and variability among the different reagents due to the labelling processes or labels themselves. On a scientific research basis, the use of these labels can also introduce difficulties such as concerns with effects on protein functionality/structure due to the presence of the attached labels and the inability to directly measure the interactions in real time.

Presented here is the use of a new label-free optical biosensor that is amenable to microarray studies, termed the Interferometric Reflectance Imaging Sensor (IRIS), for detecting proteins, DNA, antigenic material, whole pathogens (virions) and other biological material. The IRIS system has been demonstrated to have high sensitivity, precision, and reproducibility for different biomolecular interactions [1-3]. Benefits include multiplex imaging capacity, real time and endpoint measurement capabilities, and other high-throughput attributes such as reduced reagent consumption and a reduction in assay times. Additionally, the IRIS platform is simple to use, requires inexpensive equipment, and utilizes siliconbased solid phase assay components making it compatible with many contemporary surface chemistry approaches.

Here, we present the use of the IRIS system from preparation of probe arrays to incubation and measurement of target binding to analysis of the results in an endpoint format. The model system will be the capture of target antibodies which are specific for human serum albumin (HSA) on HSA-spotted substrates.

\section{Video Link}

The video component of this article can be found at https://www.jove.com/video/2694/

\section{Protocol}

\section{Substrate Preparation}

1. Coat layered silicon-SiO${ }_{2}$ substrates with self-adsorbing copoly(DMA-NAS-MAPS): 
1. Copolymer synthesis, chemical structure, and coating process are published in: G. Pirri, F. Damin, M. Chiari, E. Bontempi, L.E. Depero. Characterization of A Polymeric Adsorbed Coating for DNA Microarray Glass Slides. Anal. Chem. 2004, 76, $1352-58$.

2. Briefly, prepare polymer solution by adding $100 \mathrm{mg}$ of polymer to $5 \mathrm{~mL}$ of deionized (DI) water and add $5 \mathrm{~mL}$ of $40 \%$ saturated ammonium sulfate $\left(\left(\mathrm{NH}_{4}\right) 2 \mathrm{SO}_{4}\right)$ solution to reach a final concentration of $0.92 \mathrm{M}$.

3. Submerge chips in solution for $30 \mathrm{~min}$ on a shaker and then rinse thoroughly with DI water. Dry thoroughly with $\mathrm{Argon} / \mathrm{N}_{2}$ gas.

4. Bake chips at $80^{\circ} \mathrm{C}$ for $15 \mathrm{~min}$.

2. Store polymer-coated substrates/chips in a dry environment (vacuum desiccator) for up to 3 months until probe spotting procedure.

\section{Preparation of Probe Array: Antibodies, antigens, ss/dsDNA, RNA, etc.}

1. Dilute probe(s) to appropriate concentration in desired buffer. This step can be vary considerably, but a typical experiment utilizes antigen or IgG at a concentration of $0.5 \mathrm{mg} / \mathrm{mL}$ (range of $0.1-1 \mathrm{mg} / \mathrm{mL}$ ) in phosphate buffered saline (PBS) at pH $\approx .4$.

2. Place solutions in a 96 - or 384 -well plate (or standard source plate for the spotter being used)

3. Setup spotting parameters for desired printed array: determine the appropriate spotting parameters for the surface and solutions being used (dwell times, approach speeds, etc.). Determine the number of replicates of each condition per grid, the grid layout, the number of replicate grids, and the desired spotting location on the chip. Place substrates and source plate in the appropriate locations, check to make sure waste and supply bottles are ready, and begin printing run.

4. After spotting is finished place substrates in a high humidity environment overnight (4-18 hours) to allow immobilization and deactivation process to proceed.

5. Wash substrates: place them in the following solutions for three minutes for three separate washes: PBS with $0.1 \%$ Tween (PBST), PBS, and finally deionized (DI) water. Depending on the type of experiment (wet vs. dry), dry the chip thoroughly with Argon gas and begin incubation or place the chip in a flow chamber and assemble.

6. Deactivate remaining NHS groups on copolymer surface by submerging the chips in a $50 \mathrm{mM}$ solution of ethanolamine with the pH adjusted to 7.4 for 30 minutes while on a shaker plate. A primary amine-containing compound such as hydroxylamine or Tris can also be used, as long the prepared solution is safe to use with proteins. Thoroughly rinse the deactivation solution using PBS then DI water.

7. Optional step (depending on the surface chemistry being employed): Block surface using a solution of BSA, casein, or rehydrated milk for 30 minutes. For the current polymeric surface chemistry that we use, we do not perform this step as the polymer backbone acts to prevent nonspecific protein binding.

\section{Data Acquisition and Incubation Procedure: Endpoint format}

1. Make a solution of the target of interest in the desired buffer. Here, it will be a solution of the appropriate concentration of Anti-BSA in PBS (ex: make $1-10 \mathrm{~mL}$ of a $10 \mathrm{ng} / \mathrm{mL}$ solution of Anti-BSA in PBS). Also, make sure to have an equivalent amount of buffer (without target) for the negative control incubation.

2. Take a silicon mirror scan for normalizing all subsequently collected data.

3. Scan the prepared probe array with the IRIS system prior to the incubation step to determine the initial optical height (mass) at each spot. This will allow for proper analysis of changes in mass on the surface, i.e. the level of binding, after the incubation. Here, a few parameters will be adjusted for the current experiment, such as exposure time, field of view, prospering focusing, etc.

4. Submerge chip(s) in prepared solution for 1 hour on a shaker plate. The incubation time can vary considerably depending on the level of agitation, the concentration of target being detected, the transport characteristics of the flow chamber (if a real-time detection mode is being used), the affinities of the target-probe pair, etc.

5. After the incubation, repeat the wash procedure described in step 2.5)

6. Scan the same probe array using the IRIS system and obtain post-incubation images for pre-incubation comparison.

7. Repeat this process for different incubation steps if necessary, for example in a sandwich assay format where a secondary antibody is being used.

\section{Data Analysis}

1. Fit the collected images for each IRIS scan (sequence of intensity images), using the lab-developed software to produce an image of the probe array containing optical thickness information. A quick qualitative analysis of binding can be performed by subtracting (registered) postand pre-incubation images. Binding will appear as a change in intensity at those spots where mass changes were observed. For quantitative analysis, determine mass densities of each spot compared to the background by averaging optical thickness information for each pixel within a spot and an annulus outside of the spot and making a direct comparison of these two areas.

\section{Representative Results:}

Figure 1 shows an example schematic of the layered $\mathrm{Si}_{-} \mathrm{SiO}_{2} \mathrm{IRIS}$ substrate spotted with a representative antibody array. Each antibody ensemble is spotted in replicate with specificity for a different epitope targeting different proteins. Two different whole viruses and a viral protein are represented as example targets. Negative control antibodies depend on the assay and can be non-specific and/or virus-specific.

Figure 2 shows the binding of human serum albumin (HSA)-specific antibodies to an array of spotted HSA and rabbit IgG (control) spots. As seen here, after fitting and determination of the optical thicknesses for the pre- and post-incubation images, a difference image for the binding array can be created to qualitatively assess binding. Quantitative analysis reveals that a 2.05 and 0.13 nanometer mean optical height change was observed for the HSA and rabbit lgG spots, respectively, for an anti-HSA incubation concentration of $500 \mathrm{ng} / \mathrm{mL}$.

Figure 3 shows an IRIS measurement of Fur protein binding dependence on protein concentration and dsDNA oligomer length. The top graph shows absolute optical height measurements for initial immobilized oligomer probe heights and post-incubation Fur binding. Increasing 
concentrations of Fur $(50,100,200 \mathrm{nM})$ resulted in increased binding to DNA probes. The length of the oligomers also impacts Fur binding with longer sequences resulting in more binding. Here, error bars represent one standard deviation from the mean. The bottom plot shows calculated Fur protein dimer binding per dsDNA strand. Dimer binding was significantly increased at a Fur protein concentration of $200 \mathrm{nM}$ suggesting a high-level of non-specific binding.

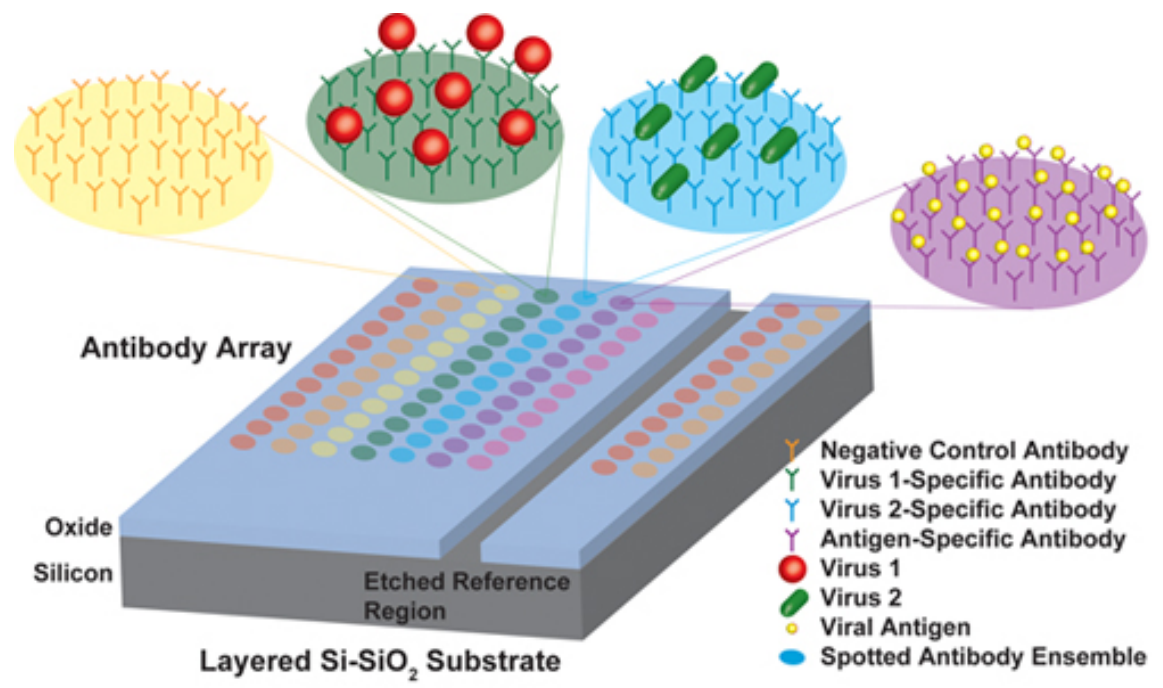

Figure 1. Schematic of an example probe array normally used in an experiment to detect specific viruses and viral components in a multiplexed fashion with the IRIS system.

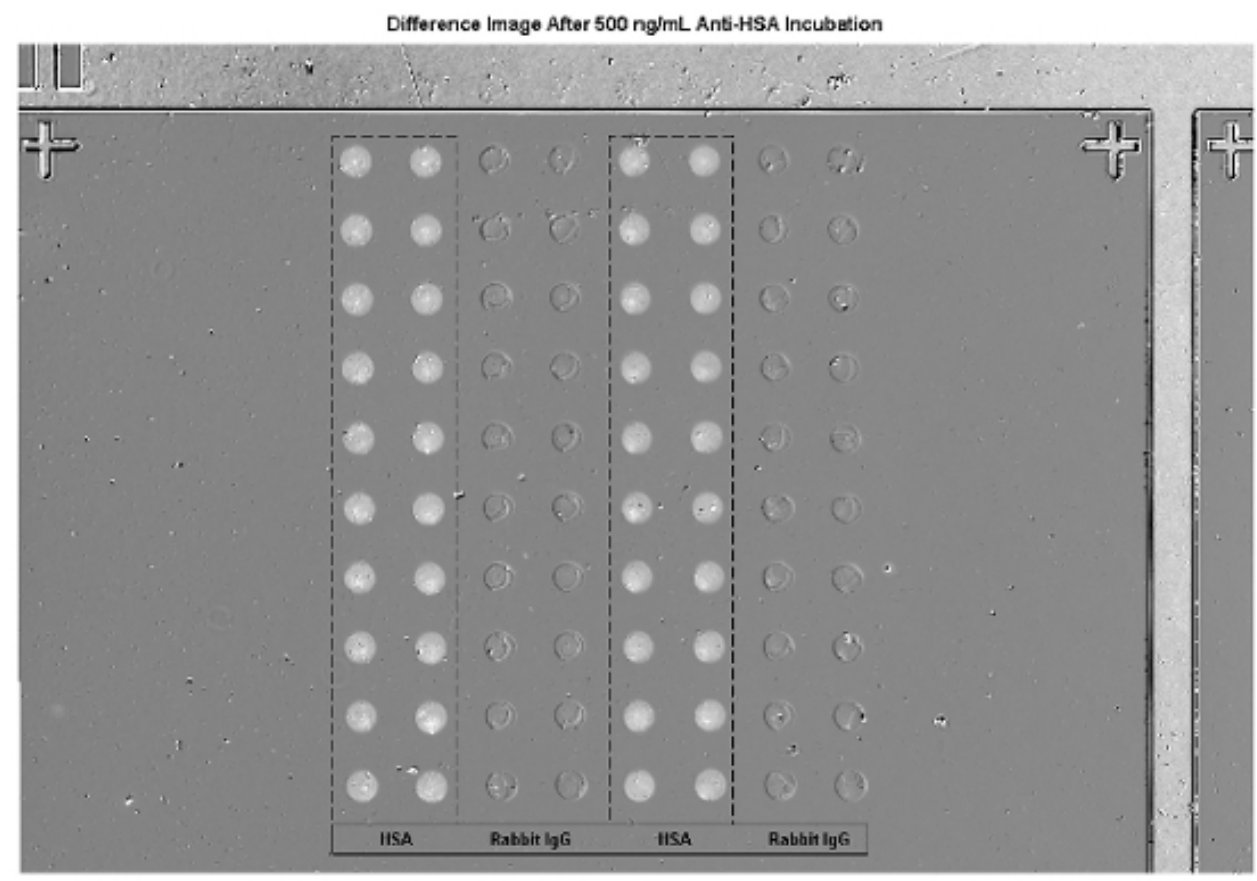

Spotted Probe Condition

Figure 2. Post-incubation difference image for binding of human serum albumin-specific antibodies to spotted HSA collected with this label-free system at a concentration of $500 \mathrm{ng} / \mathrm{mL}$. The biomaterial mass density for each spot is determined by averaging optical thickness information within a spot and then comparing this with the average of an annulus around the spot representing the background. 

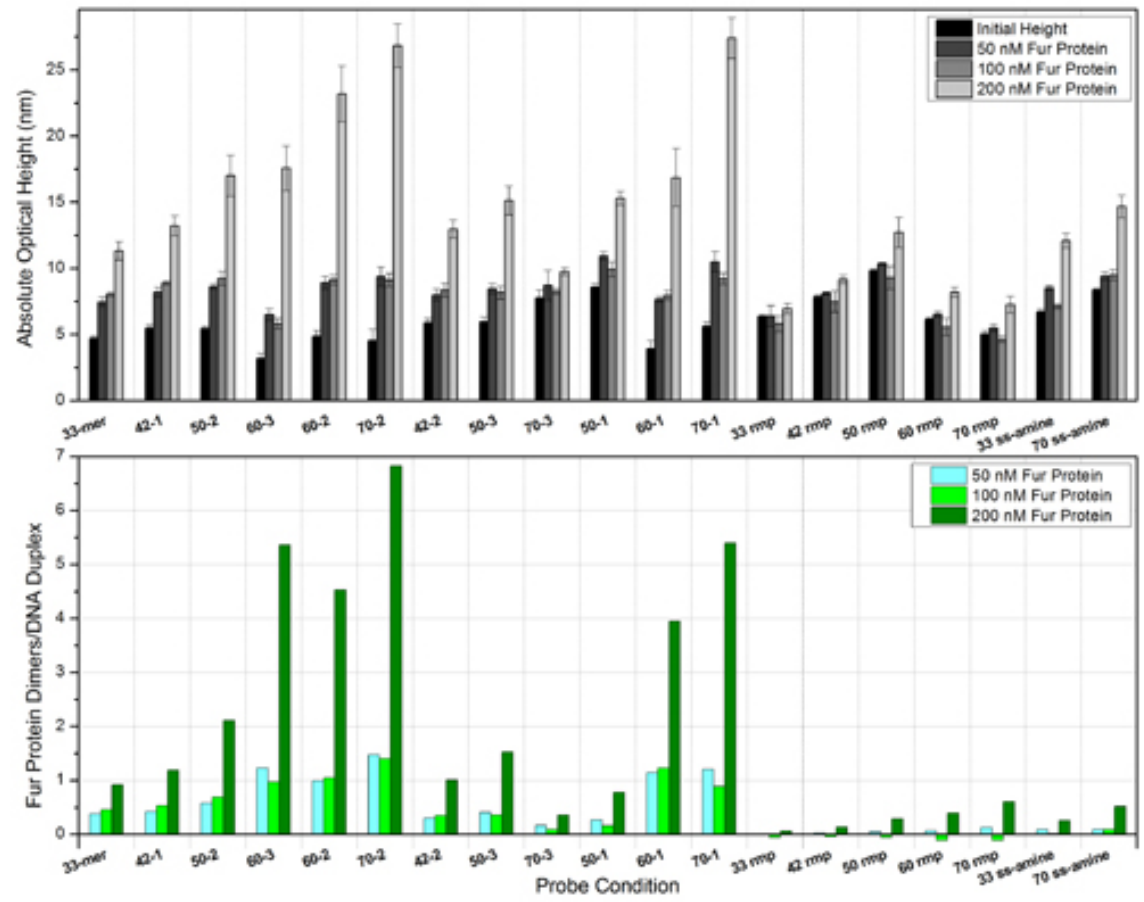

Figure 3. Plot of binding data for Fur protein interactions with spotted double-stranded oligomers with a known consensus sequence based on oligomer length, location of the consensus sequence within the oligomer, and Fur protein concentration. Information about the absolute amount of Fur protein bound to each spotted sequence (top) can be used to estimate the number of Fur dimers bound to each type of oligomer (bottom).

\section{Discussion}

The IRIS platform is a simple and rapid system to use for collecting high-throughput binding data in a microarray format. By covalently immobilizing different functional protein or DNA probes on a surface, target antigens/biomarkers/etc. can be captured from solution, as in an immunoassay. Measurement of these interactions across probe conditions in an endpoint or real-time format with the IRIS system allows for highly sensitive and quantitative information to be collected for each interaction. The representative experiment detailed here is just one example of the types of interactions that can be studied with this technique. Detection of transcription factors, viral antigens, and whole viruses has been demonstrated previously and represents just a few examples of the types of analyses that the IRIS can easily handle.

\section{Disclosures}

No conflicts of interest declared.

\section{Acknowledgements}

The authors wish to thank Zoiray Technologies Inc., a co-sponsor and commercialization partner, for its support.

\section{References}

1. Özkumur, E., Needham, J.W., Bergstein, D.A., Gonzalez, R., Cabodi, M., Gershoni, J.M., Goldberg, B.B. and Ünlü, M.S. Label-free and dynamic detection of biomolecular interactions for high-throughput microarray applications. Proceedings of the National Academy of Sciences. 105 (23) : 7988-92 (2008).

2. Özkumur, E., Ahn, S., Yalcin, A., Lopez, C.A., Cevik, E., Irani, R.J., DeLisi, C., Chiari, M. and Ünlü, M.S. Label-free microarray imaging for direct detection of DNA hybridization and single-nucleotide mismatches. Biosensors and Bioelectronics. 25 (7): $1789-95$ (2010).

3. Özkumur, E., Lopez, C.A., Yalcin, A., Bergstein, D.A., Connor, J.H., Chiari, M., Ünlü, M.S. Spectral reflectance imaging for a multiplexed high-throughput, label-free, and dynamic biosensing platform. IEEE Journal of Selected Topics in Quantum Electronics. 16 (3) : $635-46$ (2010).

4. Lopez, C.A., Daaboul, G.G., Vedula, R.S., Özkumur, E., Bergstein, D.A., Geisbert, T.W., Fawcett, H.E., Goldberg, B.B., Connor, J.H., Ünlü, M.S. Label-free multiplexed virus detection using spectral reflectance imaging. Biosensors and Bioelectronics. doi: 10.1016/ j.bios.2011.01.019 (2011). 